\title{
Our panel of experts highlight the most important research articles across the spectrum of topics relevant to the field of hematologic oncology
}

Expert panel: Stefano Molica, Department of Hematology-Oncology, Azienda Ospedaliera Pugliese-Ciaccio, Catanzaro, Italy; Dominic Chamberlain, Future Medicine Ltd, London, UK

\begin{abstract}
Hillmen P, Gribben JG, Follows GA et al. Rituximab plus chlorambucil as firstline treatment for chronic lymphocytic leukemia: final analysis of an open-label Phase II study. J. Clin. Oncol. doi:10.1200/ JCO.2013.49.6547 (2014) (Epub ahead of print).
\end{abstract}

In this Phase II study, the authors evaluated the safety and efficacy of rituximab (R) in combination with chlorambucil (R-chlorambucil) in previously untreated chronic lymphocytic leukemia (CLL) patients who cannot tolerate $\mathrm{R}$ in association with fludarabine and cyclophosphamide. All patients had progressive Binet stage B or stage C CLL. Treatment consisted of $\mathrm{R}\left(375 \mathrm{mg} / \mathrm{m}^{2}\right.$ on day 1 , cycle 1 and $500 \mathrm{mg} / \mathrm{m}^{2}$ thereafter) plus chlorambucil $\left(10 \mathrm{mg} / \mathrm{m}^{2} /\right.$ day all cycles; day 1 through to 7) for six 28-day cycles. Assessment of response was performed according to NCI revised guidelines for CLL after cycle three and cycles six. For patients not achieving complete response, six additional cycles of chlorambucil alone could be administered.

A total of 100 patients with median age 70 years (range: $43-86$ ) were enrolled in this multicenter study. The overall response rate was $84 \% ; 10 \%$ of patients achieved confirmed complete remission and $74 \%$ achieved partial remission. In addition, 48 patients had their response confirmed by CT scan. Median duration of response was 21.2 months and median progression-free survival was 23.5 months. Median overall survival was not reached, and after 30 months of follow-up, 84 patients were still alive.

Results of this study suggest that first-line chlorambucil combined with an anti-CD20 monoclonal antibody is an effective option in patients not eligible for $\mathrm{R}$ in association with fludarabine and cyclophosphamide. These data are in keeping with those presented at the last American Society of Hematology meeting with an association of chlorambucil and different newer anti-CD20 monoclonal antibodies (i.e., obinutuzumab and ofatumumab) [1-3]. In these two randomized clinical trials the efficacy of adding an anti-CD20 monoclonal antibody to chlorambucil was evident in terms of progression-free survival $[1,2]$. All these studies establish chemoimmunotherapy as the current treatment of choice for all CLL patients, and demonstrate the feasibility of enrolling patients that better reflect the age group of patients with CLL into clinical trials.

- Written by S Molica

Zhou Z, Sehn LH, Rademaker AW. An enhanced International Prognostic Index (NCCN-IPI) for patients with diffuse large B-cell lymphoma treated in the rituximab era. Blood 123(6), 837-842 (2014).

The International Prognostic Index (IPI) is a well-recognized tool for assessing prognosis of patients with diffuse large B-cell lymphoma (DLBCL). Five clinical characteristics including age, $\mathrm{LDH}$, number of extranodal sites, Ann Arbor stage, and Eastern Cooperative Oncology Group (ECOG) performance status are included in the IPI score and lead to patients' risk stratification into four discrete risk categories. However, IPI score was developed more than 20 years ago on the basis of the clinical characteristics of patients with DLBCL treated with cyclophosphamide, doxorubicin, vincristine and prednisone (CHOP) or CHOP-like chemotherapy.
Future
Medicine 
In a recent issue of Blood, Zhou and colleagues present an advanced prognostic index for DLBCL, based on the analysis of patients treated in the rituximab era.

The authors reviewed clinical features of 1650 adults with de novo DLBCL diagnosed in the period 2000-2010 at seven National Cancer Center Netwowork (NCCN) centers with the effort to further refine the categorization of age and normalized LDH. Five predictors (age, $\mathrm{LDH}$, sites of involvement, Ann Arbor stage and ECOG performance status) were identified and a maximum of eight points assigned. Four risk groups were recognized: low $(0-1)$, low-intermediate (2-3), high-intermediate (4-5) and high (6-8). Compared with the IPI, the NCCN-IPI better discriminated low- and high-risk subgroups (5-year overall survival: 96 vs $33 \%$ ) than the IPI (5-year overall survival: $90 \%$ vs $54 \%$ ), respectively.

From a practical standpoint, the NCCNIPI can be easily applied in academic and community-based practice settings alike.

On the other hand, the authors successfully validated NCCC-IPI using an independent cohort from the British Columbia Cancer Agency $(\mathrm{n}=1138)$.

It is not clear whether the NCCN-IPI will retain its prognostic value for risk stratification in the context of novel targeted therapies. What we expect is that biologic markers such as geneexpression-based signatures and molecular biomarkers should be evaluated for their capacity to improve upon the NCCN-IPI [4].

- Written by S Molica

Shah N, Guilhot F, Cortes J et al. Long-term outcome with dasatinib after imatinib failure in chronic-phase chronic myeloid leukemia: follow-up of a Phase 3 study. Blood 123, 2317-2324 (2014).

In this study the authors present results from a long-term follow-up of a dasatinib Phase II study of patients with imatinib-resistant/-intolerant chronic myeloid leukemia (CML). The original Phase III study, consisted of a total of 670 patients with imatinib-resistant/-intolerant CML in chronic phase receiving dasatinib 100 $\mathrm{mg}$ once daily, $50 \mathrm{mg}$ twice daily, $140 \mathrm{mg}$ once daily or $70 \mathrm{mg}$ twice daily. After 6 years, 188 $(28 \%)$ of the original 670 patients remained on study treatment. Estimated 6-year protocoldefined progression-free survival (PFS) rates were 49, 51, 40 and 47\%, respectively, and estimated 6-year overall survival rates were 71 , 74, 77 and 70\%, respectively. Estimated 6-year rates of survival without transformation on study treatment were $76,80,83$ and $74 \%$, respectively. Major molecular response was achieved in $43 \%$ (100 mg once daily) and 40\% (all other arms) of patients by 6 years. Molecular and cytogenetic responses at 3 and 6 months were highly predictive of PFS and overall survival. Estimated 6 -year PFS rates based on $\leq 1 \%,>1 \%$ to $10 \%$ and $>10 \%$ BCR-ABL transcripts at 3 months were 68, 58 and 26\%, respectively. Most adverse events occurred by 2 years. The authors argue that these results indicate that a consistent subgroup of CML chronic phase resistant or intolerant patients can have a long-term benefit from dasatinib therapy, in particular those with faster and deeper responses to dasatinib.

- Written by D Chamberlain

Tuscano J, Dutia M, Chee K et al. Lenalidomide plus rituximab can produce durable clinical responses in patients with relapsed or refractory, indolent non-Hodgkin lymphoma. Br. J. Haematol. 165(3), 375-381 (2014).

In this Phase II study the authors evaluated the safety and efficacy of lenalidomide in combination with rituximab in patients with relapsed/refractory, indolent non-Hodgkin lymphoma (NHL). Patients were treated with daily lenalidomide in 28-day cycles with weekly rituximab for 4 weeks. Lenalidomide was continued until progression or unacceptable toxicity. A total of 22 patients were assessed for FCGR3A polymorphisms. A total of 30 patients were enrolled; 27 were evaluable for response. The overall response rate (ORR) was $74 \%$ including $44 \%$ complete responses, with median progression-free survival reported as 12.4 months. The ORR of the 13 rituximab refractory patients and in the in the 22 follicular lymphoma patients was 61.5 and $77 \%$, respectively. At a follow-up time of 43 months, the median duration of response and time to next therapy were 15.4 and 37.4 months, respectively. Most common grade $3 / 4$ adverse events were lymphopenia (45\%), neutropenia $(55 \%)$, fatigue $(23 \%)$ and hyponatremia (9\%). The authors report that the ORR and progression-free survival in patients with lowaffinity FCGR3A polymorphisms suggest that 
lenalidomide may improve the activity of rituximab in these patients. The authors conclude that these data suggest that combining lenalidomide with rituximab can produce durable responses with acceptable toxicity in patients with indolent non-Hodgkin lymphoma.

- Written by D Chamberlain

Financial \& competing interests disclosure

$D$ Chamberlain is an employee of Future Medicine Ltd. The authors have no other relevant affiliations or financial involvement with any organization or entity with a financial interest in or financial conflict with the subject matter or materials discussed in the manuscript apart from those disclosed.

No writing assistance was utilized in the production of this manuscript.

\section{References}

1 Molica S. Highlights in the treatment of chronic lymphocytic leukemia from the 2013 meeting of the American Society of Hematology. Expert Rev. Hematol. 7(2), 187-190 (2014).

2 Goede V, Fischer K, Busch R et al. Obinutuzumab plus chlorambucil in patients with CLL and coexistingconditions. N. Engl. J. Med. 370(12), 1101-1110 (2014).

3 Hillmen P, Robak T, Janssens A et al. Ofatumumab + chlorambucil versus chlorambucil alone in patients with untreated chronic lymphocytic leukemia (CLL): results of the Phase III study complement 1 (OMB110911). Presented at: 55th American Society of Hematology Annual Meeting and Exposition. New Orleans, LA, USA, 7-10 December 2013 (Abstract 528).

4 Kanakry JA, Ambinder RF. Old variables, new value: a refined IPI for DLBCL Blood 123, 800-801 (2014). 